\title{
On the rational monodromy-free potentials with sextic growth
}

\author{
J.Gibbons \\ Imperial College, 180 Queen's Gate, \\ London SW7 2BZ \\ j.gibbons@ic.ac.uk
}

\author{
A.P. Veselov \\ Department of Mathematics, Loughborough University, \\ Loughborough, LE11 3TU \\ and \\ Landau Institute for Theoretical Physics, \\ Moscow, Russia \\ A.P.Veselov@lboro.ac.uk
}

October 28, 2018

\begin{abstract}
We study the rational potentials $V(x)$, with sextic growth at infinity, such that the corresponding one-dimensional Schrödinger equation has no monodromy in the complex domain for all values of the spectral parameter. We investigate in detail the subclass of such potentials which can be constructed by the Darboux transformations from the well-known class of quasi-exactly solvable potentials

$$
V=x^{6}-\nu x^{2}+\frac{l(l+1)}{x^{2}} .
$$

We show that, in contrast with the case of quadratic growth, there are monodromy-free potentials which have quasi-rational eigenfunctions, but which can not be given by this construction. We discuss the relations between the corresponding algebraic varieties, and present some elementary solutions of the Calogero-Moser problem in the external field with sextic potential.
\end{abstract}




\section{Introduction}

Consider the Schrödinger equation with a meromorphic potential $V(x)$ :

$$
\left(-D^{2}+V(x)\right) \psi=\lambda \psi
$$

where here and below $D=\frac{\mathrm{d}}{\mathrm{d} x}$. We say that such a potential $V(x)$ has trivial monodromy, if all the solutions $\psi$ of the corresponding equation (11) are meromorphic in $x$ for all $\lambda$. The general problem is to describe all such potentials.

In such generality this is probably a hopeless question. However if we restrict ourselves to a suitable class of potentials it is known to be solvable. The first results in this direction were found by Duistermaat and Grünbaum [1], who solved it in the class of rational potentials decaying at infinity:

$$
V=\sum_{i=1}^{N} \frac{k_{i}\left(k_{i}+1\right)}{\left(x-x_{i}\right)^{2}} .
$$

They showed that the corresponding parameters $k_{i}$ must be integers, and all such potentials are the results of Darboux transformations applied to the zero potential. Therefore they are given by the Burchnall-Chaundy (or Adler-Moser) explicit formulas [2, 3]. The corresponding configurations of the poles $x_{i}$ are very special: in the case when all the parameters $k_{i}=1$, they are nothing else but the (complex) equilibria of the Calogero-Moser system, with the Hamiltonian

$$
H=\sum_{i=1}^{N} p_{i}^{2}+\sum_{j \neq i}^{N} \frac{2}{\left(x_{i}-x_{j}\right)^{2}},
$$

which are described by the following algebraic system:

$$
\sum_{j \neq i}^{N} \frac{1}{\left(x_{i}-x_{j}\right)^{3}}=0, \quad i=1, \ldots, N .
$$

A remarkable fact (discovered first by Airault, McKean and Moser [4]) is that this system has no solutions unless

$$
N=\frac{m(m+1)}{2}
$$

is a triangular number, in which case the solutions depend on $m$ arbitrary complex parameters. 
Oblomkov [5] generalised the Duistermaat-Grünbaum result to the case of rational potentials with quadratic growth at infinity:

$$
V=x^{2}+\sum_{i=1}^{N} \frac{k_{i}\left(k_{i}+1\right)}{\left(x-x_{i}\right)^{2}},
$$

by proving that all such potentials can be found by applying successive Darboux transformations to the harmonic oscillator, and that they have the following explicit description in terms of the Wronskians of Hermite polynomials $H_{k}(x)$ :

$$
V=x^{2}-2 D^{2} \log W\left(H_{k_{1}}, H_{k_{2}}, \ldots, H_{k_{n}}\right) .
$$

In particular the corresponding Schrödinger equations must have infinitely many solutions of the quasi-rational form

$$
\psi=R(x) \exp \left( \pm x^{2} / 2\right)
$$

where $R(x)$ is some rational function.

An interpretation of this result in terms of the generalised Stieltjes relations and the rational solutions of the dressing chain [7] was proposed in the paper [6], where also a new class of meromorphic monodromy-free potentials, expressible in terms of the Painleve transcendents, was found.

In this paper we consider the set $\mathbf{M}$ of monodromy-free even rational potentials with sextic growth at infinity:

$$
V=P_{6}(x)+\sum_{i=1}^{N} \frac{k_{i}\left(k_{i}+1\right)}{\left(x-x_{i}\right)^{2}}
$$

where $P_{6}(x)$ is an even polynomial of degree 6 , which for simplicity will be assumed to be of the form

$$
P_{6}(x)=x^{6}-\nu x^{2}
$$

where $\nu$ is a parameter.

Our motivation came from the theory of Calogero-Moser systems. It is known, after Inozemtsev [8], that the corresponding classical system in an external field with the Hamiltonian:

$$
H=\sum_{i=1}^{N} p_{i}^{2}+\sum_{i=0}^{N} P_{6}\left(x_{i}\right)+\sum_{j \neq i}^{N} \frac{1}{\left(x_{i}-x_{j}\right)^{2}}
$$

is integrable (note that this is believed to be not the case if the degree of $P$ is higher than 6), so one can expect some effective way to describe the equilibria of this system and the corresponding set $\mathbf{M}$. 
Having in mind the case of quadratic growth, let us consider also the set $\mathbf{S}$ of potentials (4), such that the corresponding Schrödinger operator has a quasi-rational eigenfunction, of the form

$$
\psi=R(x) \exp \left( \pm x^{4} / 4\right)
$$

where $R(x)$ is some rational function.

Finally we consider the set $\mathbf{D}$ of the operators, which are Darboux-related to the operators of the form

$$
\mathcal{L}_{p, m}=-D^{2}+x^{6}-(2 p+1) x^{2}+\frac{m(m+1)}{x^{2}}
$$

with integer $p$ and $m$. The operators $\mathcal{L}_{p, m}$ are known, after Turbiner, [9, 10], to be quasi-exactly solvable, and they were studied later from various points of view in [11, 12, 13, 14, 15, 16]. They have $M$ quasi-rational eigenfunctions

$$
\psi_{i}^{(p, m)}=R_{i}^{(p, m)}(x) \exp \left( \pm x^{4} / 4\right), \quad i=1, \ldots, M,
$$

where $M$ is a positive integer determined by $p$ and $m$ (see section 2 below). The set $\mathbf{D}$ consists of the potentials of the form:

$$
V(x)=x^{6}-(2 p+1) x^{2}+\frac{m(m+1)}{x^{2}}-2 D^{2} \log W_{I}(x),
$$

where $I \subset\{1,2, \ldots, M\}$ and $W_{I}$ is the Wronskian of the corresponding quasi-rational eigenfunctions $\psi_{i}, i \in I$.

We will study all these three sets and the corresponding affine algebraic varieties. In particular, we show that, under some assumptions,

$$
\mathrm{D} \subset \mathrm{S} \subset \mathrm{M},
$$

and that, in contrast to the quadratic case, all the inclusions are proper:

$$
\mathbf{D} \neq \mathbf{S} \neq \mathbf{M}
$$

even if we restrict the definition of the set $\mathbf{M}$ to odd integer values of the parameter $\nu$. This means that only a proper subset of monodromy-free potentials with sextic growth at infinity have an explicit description in terms of Darboux transformations.

We study also the corresponding time-dependent Schrödinger equation, and show that the Stieltjes relations for them can be considered as Bäcklund transformations between Calogero-Moser systems in different sextic potentials, with different numbers of particles. We construct a family of explicit solutions in elementary functions for such a system. We note that these solutions are very special - Inozemtsev's general solution of the system is highly transcendental, being expressed in terms of Riemann $\theta$-functions. 


\section{The locus conditions}

In this section we recall the trivial monodromy conditions [1], which we will call locus equations following the terminology of [4].

Suppose that a Schrödinger equation with a rational potential $V(x)$

$$
\left(-D^{2}+V(x)\right) \psi=\lambda \psi
$$

has all its solutions meromorphic for all $\lambda$. At a singular point, which we may take to be $x=0$, we have the two series:

$$
\begin{gathered}
V(x)=x^{-D} \sum_{n=0}^{\infty} V_{n} x^{n}, \\
\psi(x, \lambda)=x^{-m} \sum_{n=0}^{\infty} \psi_{n} x^{n} .
\end{gathered}
$$

where we suppose $D, m>0$, and $\psi_{0} \neq 0$. Then the Schrödinger equation becomes:

$$
\begin{array}{r}
\left(-D^{2}+\sum_{n=0}^{\infty} V_{n} x^{n}\right) \sum_{l=0}^{\infty} \psi_{l} x^{l-m} \\
=\lambda x^{-m} \sum_{n=0}^{\infty} \psi_{n} x^{n} .
\end{array}
$$

Expanding, we find:

$$
\begin{array}{r}
-\frac{1}{2} \sum_{n=-(m+2)}^{\infty}(n+2)(n+1) \psi_{n+2} x^{n}+x^{-(m+D)} \sum_{n=0}^{\infty} x^{n} \sum_{l=0}^{n} \psi_{l} V_{n-l} \\
=\lambda x^{-m} \sum_{n=0}^{\infty} \psi_{n} x^{n} .
\end{array}
$$

Hence, to balance the leading terms, it follows that $V(x)$ has only double poles, $D=2$, and that $V_{-2}=\frac{1}{2} m(m+1)$. The integer $m$ will be called the multiplicity of the pole.

There are further constraints. The coefficient of $x^{n}$ includes the term

$$
\frac{1}{2}(m(m+1)-n(n-1)) \psi_{n}
$$

and other terms which involve $\psi_{l} V_{n-l}$, for $l<n$. Hence the $m+1$ st equation does not involve $\psi^{m+1}$. Thus the Schrödinger equation can be solved to all 
orders, provided that the $(m+1)$ st equation is consistent with the previous equations. The resulting system may be written in matrix form:

$$
\left(\begin{array}{cccccc}
0 & \ldots & \ldots & \ldots & m & V_{1} \\
0 & \ldots & \ldots & 2 m-1 & V_{1} & V_{2}-\lambda \\
0 & \ldots & \ldots & \ldots & \ldots & \ldots \\
0 & \ldots & m l-l(l-1) / 2 & V_{1} & \ldots & V_{l} \\
0 & \ldots & \ldots & \ldots & \ldots & \ldots \\
0 & 2 m-1 & V_{1} & V_{2}-\lambda & \ldots & V_{2 m-1} \\
m & V_{1} & V_{2}-\lambda & \ldots & & V_{2 m} \\
V_{1} & V_{2}-\lambda & \ldots & & V_{2 m} & V_{2 m+1}
\end{array}\right)\left(\begin{array}{c}
\psi_{2 m} \\
\psi_{2 m-1} \\
\cdot \\
\cdot \\
\psi_{1} \\
\psi_{0}
\end{array}\right)=\left(\begin{array}{c}
0 \\
0 \\
\cdot \\
\cdot \\
0 \\
0
\end{array}\right)
$$

The $(2 m+1) \times(2 m+1)$ matrix on the left must be singular for all values of $\lambda$; it follows from this that the leading $m+1$ odd coefficients of the Laurent series of $V$ all vanish:

$$
V_{1}=V_{3}=\ldots=V_{2 m+1}=0 .
$$

In the special case of potentials of the form

$$
V=P(x)+\sum_{i=1}^{N} \frac{k_{i}\left(k_{i}+1\right)}{\left(x-x_{i}\right)^{2}}
$$

where $P(x)$ is some polynomial, we have an algebraic system of $K=\sum_{j=1}^{N} k_{j}$ equations in $N$ variables $x_{i}$ :

$$
P^{(2 s-1)}\left(x_{i}\right)-(2 s) ! \sum_{j \neq i}^{N} \frac{k_{j}\left(k_{j}+1\right)}{\left(x_{i}-x_{j}\right)^{2 s+1}}=0, \quad i=1, \ldots, N, s=1, \ldots k_{i} .
$$

If all the multiplicities $k_{i}=1$ we have $N$ equations for $N$ variables:

$$
P^{\prime}\left(x_{i}\right)-\sum_{j \neq i}^{N} \frac{2}{\left(x_{i}-x_{j}\right)^{3}}=0, \quad i=1, \ldots, N .
$$

In our case, with $P(x)=x^{6}-\nu x^{2}$, we will consider another special case, when $x=0$ may have an arbitrary multiplicity $l$, all other poles have multiplicity 1 , and the potential is even:

$$
V(x)=x^{6}-\nu x^{2}+\frac{l(l+1)}{x^{2}}+\sum_{i=1}^{N} \frac{2}{\left(x-x_{i}\right)^{2}},
$$

where $x_{1}, \ldots, x_{N}, N=2 n$ is a set of distinct non-zero complex numbers symmetric with respect to the origin. In that case the locus equations at 
$x=0$ are automatically satisfied, and we have the following algebraic system on $x_{i}$ :

$$
\sum_{j \neq i}^{N} \frac{2}{\left(x_{i}-x_{j}\right)^{3}}+\frac{l(l+1)}{x_{i}^{3}}+\nu x_{i}-3 x_{i}^{5}=0, \quad i=1, \ldots, N
$$

This is an affine algebraic variety $\mathbf{M}^{(N)}(\nu, l)$, which is non-empty for all values of $N$ and the parameter $\nu$.

What we are going to show here is that, for special values of the parameter $\nu$, there is a subset of such equilibria which can be described quite explicitly. They correspond to a special subclass of the monodromy-free potentials (41), and form some zero-dimensional algebraic varieties, whose properties we would like to study.

\section{The Darboux transformation}

We recall here the classical procedure going back to Darboux [17].

Let us consider the Schrodinger operator $L^{(0)}$ with potential $V^{(0)}(x)$, its eigenfunctions $\psi^{(0)}$, and in particular its eigenfunction $\psi_{1}^{(0)}$ with eigenvalue $\lambda_{1}$ :

$$
\left(L^{(0)}-\lambda_{1}\right) \psi^{(0)}=\left(-D^{2}+V^{(0)}(x)-\lambda_{1}\right) \psi^{(0)}=\left(\lambda-\lambda_{1}\right) \psi^{(0)} .
$$

The Schrödinger operator can be factorised as

$$
\left(-D^{2}+V^{(0)}(x)-\lambda_{1}\right)=-\left(D+v^{(0)}\right)\left(D-v^{(0)}\right) .
$$

The operator on the right must annihilate the eigenfunction $\psi_{1}^{(0)}$, so we may take $v^{(0)}=D \ln \left(\psi_{1}^{(0)}\right)$. The potential $V^{(0)}(x)$ is given by

$$
V^{(0)}(x)-\lambda_{1}=\frac{\mathrm{d} v^{(0)}}{\mathrm{d} x}+v^{(0)^{2}}
$$

Now we act on the left of (14) with the operator $\left(D-v^{(0)}\right)$. This takes any eigenfunction $\psi^{(0)}$ of $L^{(0)}$ into a new function

$$
\psi^{(1)}=\left(D-v^{(0)}\right) \psi^{(0)}
$$

which is either zero, if $\psi^{(0)}$ is a multiple of $\psi_{1}^{(0)}$, or else an eigenfunction of a new operator $L^{(1)}$. Explicitly

$$
-\left(D-v^{(0)}\right)\left(D+v^{(0)}\right)\left(D-v^{(0)}\right) \psi^{(0)}=\left(\lambda-\lambda_{0}\right)\left(D-v^{(0)}\right) \psi^{(0)} .
$$


so that

$$
\left(L^{(1)}-\lambda_{1}\right) \psi^{(1)}=-\left(D-v^{(0)}\right)\left(D+v^{(0)}\right) \psi^{(1)}=\left(\lambda-\lambda_{1}\right) \psi^{(1)} .
$$

The potential $V^{(1)}(x)$ in the transformed operator

$$
L^{(1)}-\lambda_{1}=-\left(D-v^{(0)}\right)\left(D+v^{(0)}\right)
$$

is then given by

$$
V^{(1)}(x)-\lambda_{1}=-\frac{\mathrm{d} v^{(0)}}{\mathrm{d} x}+v^{(0)^{2}}
$$

Thus

$$
V^{(1)}(x)-V^{(0)}(x)=-\frac{\mathrm{d} v^{(0)}}{\mathrm{d} x}=-\frac{\mathrm{d}^{2}}{\mathrm{~d} x^{2}} \ln \psi_{1}^{(0)} .
$$

If we now choose one of the remaining eigenvalues $\lambda_{2}$, say, then the transformed Schrödinger equation, shifted by this eigenvalue, is

$$
\left(L^{(1)}-\lambda_{1}\right) \psi^{(1)}=\left(-D^{2}+V^{(1)}(x)-\lambda_{1}\right) \psi^{(1)}=\left(\lambda-\lambda_{1}\right) \psi^{(1)} .
$$

We may now repeat this procedure indefinitely. The corresponding operators $L^{(n)}$ are related by

$$
L^{(n)} D^{(n)}=D^{(n)} L^{(0)},
$$

where the $n$-th order 'dressing operator' is given by:

$$
D^{(n)}=\left(D-v^{(n-1)}\right)\left(D-v^{(n-2)}\right) \ldots\left(D-v^{(0)}\right) .
$$

Since $D^{(n)}$ must annihilate the chosen set of eigenfunctions

$$
\left\{\psi_{1}^{(0)}, \ldots, \psi_{n}^{(0)}\right\}
$$

of $L^{(0)}$, with respective eigenvalues $\lambda_{1}, \lambda_{2} \ldots, \lambda_{n}$, we have

$$
\psi^{(n)}=D^{(n)} \psi^{(0)}=\frac{W_{n+1}\left(\psi_{1}^{(0)}, \psi_{2}^{(0)}, \ldots, \psi_{n}^{(0)}, \psi^{(0)}\right)}{W_{n}\left(\psi_{1}^{(0)}, \psi_{2}^{(0)}, \ldots, \psi_{n}^{(0)}\right)},
$$

where $W_{n}$ denotes the Wronskian of its $n$ arguments. Further, it may be shown by induction (see Crum [18]) that

$$
V^{(n)}(x)=V^{(0)}(x)-2 \frac{\mathrm{d}^{2}}{\mathrm{~d} x^{2}} \ln W_{n}\left(\psi_{1}^{(0)}, \psi_{2}^{(0)}, \ldots, \psi_{n}^{(0)}\right) .
$$

What is important for us, is that if the initial potential $V^{(0)}$ is meromorphic and has trivial monodromy, then it follows from (18) that the same is true for $V^{(n)}$ as well. We will use this fact in the next section to describe a class of monodromy-free potentials with sextic growth at infinity. 


\section{The even sextic potential}

The anharmonic oscillator in an even sextic potential, with Hamiltonian

$$
H=\frac{1}{2}\left(p^{2}+x^{6}-\nu x^{2}\right)
$$

has the special property that the corresponding system

$$
\frac{d^{2} x}{d t^{2}}=-3 x^{5}+\nu x
$$

can be solved explicitly using the elliptic Weierstrass $\wp$-functions:

$$
x(t)=\frac{1}{\sqrt{\wp\left(\sqrt{2 E}\left(t-t_{0}\right) ; g_{2}, g_{3}\right)-\frac{\nu}{6 E}}},
$$

where the invariants $g_{2}$ and $g_{3}$ are given by:

$$
\begin{array}{r}
g_{2}=\frac{\nu^{2}}{3 E^{2}} \\
g_{3}=\frac{2}{E}-\frac{\nu^{3}}{27 E^{3}},
\end{array}
$$

and the energy $E$ and starting time $t_{0}$ are the two arbitrary constants.

In contrast, the Schrödinger equation for the corresponding quantum system

$$
\left(-D^{2}+x^{6}-\nu x^{2}\right) \psi=\lambda \psi
$$

can not in general be solved exactly; instead it is at best quasi-exactly solvable in the sense of Turbiner [10].

To be more precise, let us consider the following slightly more general family of Schrödinger equations [10]

$$
\left(-D^{2}+x^{6}-\nu x^{2}+\frac{l(l+1)}{x^{2}}\right) \psi=\lambda \psi
$$

studied in detail in particular in [11, 12, 13, 14, 15, 16]. Let us look for a special class of quasi-polynomial solutions of the form:

$$
\psi=x^{\mu} P(x) \exp \left( \pm x^{4} / 4\right)
$$

where $P(x)$ is a polynomial. Let us consider first the case when

$$
\psi=x^{\mu} P(x) \exp \left(-x^{4} / 4\right)
$$


It is useful to rewrite this equation in terms of $\phi$, where $\psi=\phi(x) \exp \left(-x^{4} / 4\right)$ :

$$
-\left(D^{2}-2 x^{3} D-(\nu-3) x^{2}+\frac{l(l+1)}{x^{2}}\right) \phi=\lambda \phi .
$$

The coefficients $a_{n}$ of a series expansion for $\phi$ :

$$
\phi=x^{\mu} \sum_{n=0}^{\infty} a_{n} x^{n},
$$

satisfy the following recurrence relation

$$
[(\mu+n+2)(\mu+n+1)-l(l+1)] a_{n+2}+\lambda a_{n}+(\nu-2 n-2 \mu+1) a_{n-2}=0 .
$$

The very first equation gives

$$
\mu(\mu-1)-l(l+1)=0,
$$

which implies that either $\mu=l+1$ or $\mu=-l$. In the first case, the recurrence becomes:

$$
(n+2)(n+2 l+2) a_{n+2}+\lambda a_{n}+(\nu-2 n-2 l-1) a_{n-2}=0,
$$

in the second:

$$
(n+2)(n-2 l+1) a_{n+2}+\lambda a_{n}+(\nu-2 n+2 l+1) a_{n-2}=0,
$$

In particular, we see that the even and odd-index terms are decoupled, which is a consequence of the symmetry of the original problem. The quasipolynomial solutions exist when $\nu-2 n-2 l-1=0$ for some even positive integer $n$ : in that case the $a_{n}$ can all be set to zero for $n \geq 2 M$, where

$$
M=\frac{\nu-(2 l+1)}{4},
$$

which in that case is a positive integer. The eigenvalues $\lambda$ must satisfy the characteristic equation of the corresponding tridiagonal matrix, which is an algebraic equation (with integer coefficients if $l$ is an integer) of degree $2 M-2$. These polynomials are studied in more detail by Bender and Dunne [14] and sometimes called Bender-Dunne polynomials.

When $\mu=-l$ we have $M$ quasi-polynomial solutions (22), where

$$
M=\frac{\nu+(2 l+1)}{4}
$$


provided $M$ is a positive integer.

Similarly, the quasi-polynomial solutions of the form

$$
\psi=x^{l+1} P(x) \exp \left(x^{4} / 4\right)
$$

exist when

$$
M=\frac{-\nu-(2 l+1)}{4}
$$

is a positive integer; in that case there are $M$ such solutions. For the quasipolynomal solutions of the form

$$
\psi=x^{-l} P(x) \exp \left(x^{4} / 4\right),
$$

the same is true for

$$
M=\frac{-\nu+(2 l+1)}{4} .
$$

Summarising the above, we have:

Proposition 4.1 The Schrödinger equation (20) with integer l may have quasi-polynomial solutions of the form (21) only if $\nu$ is an odd integer. In that case the number of such solutions is $M$, which is the non-negative integer of the form

$$
M=\frac{ \pm \nu \pm(2 l+1)}{4} .
$$

The combination of the signs in this formula for $M$ determines the type of the corresponding solutions (21), where $P(x)$ must be an even polynomial of degree $2 M-2$. The corresponding eigenvalues $\lambda_{1}, \ldots, \lambda_{M}$ are distinct real algebraic numbers.

In particular, we see that such solutions exist for all odd $\nu$ except $\nu=$ $\pm(2 l+1)$.

Example. Let $l=0, \nu=7$ :

$$
L=-D^{2}+x^{6}-7 x^{2}
$$

Then $M=\frac{7+1}{4}=2$. Both signs are plus, so the corresponding quasipolynomial solutions are of the form

$$
\psi=x^{-l} P(x) \exp \left(-x^{4} / 4\right)=P(x) \exp \left(-x^{4} / 4\right), P(x)=a_{0}+a_{2} x^{2} .
$$

The characteristic equation is

$$
\left|\begin{array}{ll}
\lambda & 2 \\
4 & \lambda
\end{array}\right|=0
$$


giving $\lambda= \pm 2 \sqrt{2}$. For $\lambda=-2 \sqrt{2}$, the eigenfunction is found to be

$$
\psi_{1}=\left(\sqrt{2} x^{2}+1\right) \exp \left(-x^{4} / 4\right)
$$

which, having no zeroes, must be the ground state, while for $\lambda=2 \sqrt{2}$, the eigenfunction is

$$
\psi_{2}=\left(\sqrt{2} x^{2}-1\right) \exp \left(-x^{4} / 4\right)
$$

This has two zeroes, so there must be another (odd) excited state, with a single zero, with eigenvalue between in the interval $(-2 \sqrt{2}, 2 \sqrt{2})$ - however this eigenfunction is not quasi-polynomial.

\section{Dressing the sextic potential}

The potentials

$$
V=x^{6}-\nu x^{2}+\frac{l(l+1)}{x^{2}}
$$

with integer $l$ are clearly monodromy-free, so we can apply the Darboux transformations to construct more operators with trivial monodromy. If we use only quasi-polynomial eigenfunctions, all the new potentials will be rational with sextic growth at infinity. Indeed by the Crum formula (18) the new potential is

$$
V_{I}=V-2 D^{2} \ln W_{I}(x),
$$

where $W_{I}=W\left(\psi_{i_{1}}, \psi_{i_{2}}, \ldots, \psi_{i_{m}}\right)$ is the Wronskian of the quasi-polynomial eigenfunctions (21) from a subset $I=\left\{i_{1}, i_{2}, \ldots, i_{m}\right\} \subset\{1, \ldots, M\}$.

In the case when these eigenfunctions have the form

$$
\psi_{i}(x)=x^{l+1} P_{i}(x) \exp \left(x^{4} / 4\right)
$$

we have the following

Proposition 5.1 The Wronskian $W_{I}$ has a form

$$
W_{I}=x^{m(l+1)+\frac{1}{2} m(m-1)} P_{I}(x) \exp \left(m x^{4} / 4\right)
$$

with an even polynomial $P_{I}$ of degree

$$
2 d=2 m(M-m)
$$

non-vanishing at zero. 
Indeed we have

$$
W\left(\psi_{i_{1}}, \psi_{i_{2}}, \ldots, \psi_{i_{m}}\right)=x^{m(l+1)} \exp \left(m x^{4} / 4\right) W\left(P_{i_{1}}, P_{i_{2}}, \ldots, P_{i_{m}}\right),
$$

where $P_{i}$ are the corresponding even polynomials whose coefficients $a_{k}$ are determined recurrently by (23). We claim that

$$
W\left(P_{i_{1}}, P_{i_{2}}, \ldots, P_{i_{m}}\right)=x^{\frac{1}{2} m(m-1)} P(x)
$$

for some even polynomial $P$ with $P(0) \neq 0$. To prove this let us assume without loss of generality that $i_{k}=k$. We can apply elementary row operations to the coefficients of the polynomials $P_{1}, \ldots, P_{m}$ so that the lowest order terms are of increasing degree:

$$
\tilde{P}_{k}=x^{2 k} Q_{k}(x)
$$

for some even polynomials $Q_{k}(x)$. We claim that $Q_{k}(0) \neq 0$. For this we need to prove the independence of the corresponding vectors

$$
a^{(i)}=\left(a_{0}^{(i)}, a_{2}^{(i)}, \ldots, a_{2 k}^{(i)}\right), i=1, \ldots, k
$$

determined by the recurrence relations (24). From these relations it follows that $a_{2 j}$ is a polynomial in $\lambda$ of degree $j$ and thus $a_{2 j}^{(i)}$ are the values of these polynomials at the eigenvalues $\lambda_{i}$. Since we know that all $\lambda_{i}$ are distinct the claim now follows from the Vandermonde formula.

This implies that the leading term of the Wronskian

$$
W\left(P_{i_{1}}, \ldots, P_{i_{m}}\right)=W\left(\tilde{P}_{i_{1}}, \ldots, \tilde{P}_{i_{m}}\right)
$$

at zero is

$$
x^{0+1+\ldots+(m-1)}=x^{\frac{1}{2} m(m-1)} .
$$

Applying a similar procedure at infinity we can instead make the degrees strictly decreasing:

$$
\operatorname{deg} \tilde{P}_{i}=2 M-2 i,
$$

so the degree of the Wronskian is

$2 M-2+(2 M-5)+(2 M-8)+\ldots+(2 M-3 m+1)=m(2 M-2)-\frac{3}{2} m(m-1)$.

This means that the degree of the polynomial $P(x)$ in $(36)$ is

$$
m(2 M-2)-\frac{3}{2} m(m-1)-\frac{1}{2} m(m-1)=2 m(M-m) .
$$

This completes the proof. 

form

Thus the result of the corresponding Darboux transformations has the

$$
V_{I}=x^{6}-(\nu-6 m) x^{2}+\frac{l(l+1)+2 m(l+1)+m(m-1)}{x^{2}}-2 D^{2} \ln P_{I}(x),
$$

which is equal to

$$
V_{I}=x^{6}-(\nu-6 m) x^{2}+\frac{(l+m)(l+m+1)}{x^{2}}-2 D^{2} \ln P_{I}(x)
$$

with an even polynomial $P_{I}$ of degree $2 d=2 m(M-m)$, where

$$
M=\frac{\nu-(2 l+1)}{4} .
$$

We will denote this set of potentials $D_{+-}$having in mind the combination of signs in the formula for $M$.

Similarly we have three more sets:

$D_{++}$consisting of the results of $m$ Darboux transformations using the eigenfunctions of the form $\psi_{i}(x)=x^{-l} P(x) \exp -x^{4} / 4$ :

$$
\begin{gathered}
V_{I}=x^{6}-(\nu-6 m) x^{2}+\frac{(l-m)(l-m+1)}{x^{2}}-2 D^{2} \ln P_{I}(x), \\
\operatorname{deg} P_{I}(x)=2 m(M-m), M=\frac{\nu+(2 l+1)}{4} ;
\end{gathered}
$$

$D_{-+}$consisting of the results of $m$ Darboux transformations using the eigenfunctions of the form $\psi_{i}(x)=x^{-l} P(x) \exp x^{4} / 4$ :

$$
\begin{gathered}
V_{I}=x^{6}-(\nu+6 m) x^{2}+\frac{(l-m)(l-m+1)}{x^{2}}-2 D^{2} \ln P_{I}(x), \\
\operatorname{deg} P_{I}(x)=2 m(M-m), M=\frac{-\nu+(2 l+1)}{4} ;
\end{gathered}
$$

$D_{--}$consisting of the results of $m$ Darboux transformations using the eigenfunctions of the form $\psi_{i}(x)=x^{l+1} P(x) \exp x^{4} / 4$ :

$$
\begin{gathered}
V_{I}=x^{6}-(\nu+6 m) x^{2}+\frac{(l+m)(l+m+1)}{x^{2}}-2 D^{2} \ln P_{I}(x), \\
\operatorname{deg} P_{I}(x)=2 m(M-m), M=\frac{-\nu-(2 l+1)}{4} .
\end{gathered}
$$

However there are two natural duality relations between these sets. They correspond to the spectral equivalences between the operators (20) studied by Dorey, Dunning and Tateo in [16]. 
First of all the form of the potentials in (20) is clearly invariant under the change

$$
l \rightarrow-1-l, \nu \rightarrow \nu
$$

which means that the sets

$$
D_{++}=D_{+-}, D_{-+}=D_{--}
$$

provided we allow $l$ to be any integer.

The second duality is more interesting. Let us note that in the $D_{+-}$case, when $m=M$, the resulting potential is

$$
V_{M}=V^{*}=x^{6}-(\nu-6 M) x^{2}+\frac{(l+M)(l+M+1)}{x^{2}}
$$

which corresponds to the change

$$
\nu \rightarrow \nu^{*}=\nu-6 M, \quad l \rightarrow l^{*}=l+M .
$$

We can formally add

$$
M \rightarrow M^{*}=-M
$$

to make it an involution, which is nothing else but the "third spectral equivalence" from [16].

Note that we can allow $M$ to be a negative integer, provided we replace $M$ by $|M|$ in the formula for the degree of the polynomials $P_{I}$

$$
2 d=m(|M|-m) .
$$

This duality means that one can consider the potential (37) as a result of Darboux transformations applied to both

$$
V=x^{6}-\nu x^{2}+\frac{l(l+1)}{x^{2}}
$$

and its dual (39). In the second case one should replace the subset $I \subset$ $\{1,2, \ldots,|M|\}$ by its complement $I^{*}=\{1,2, \ldots,|M|\} \backslash I$ (and thus $m$ by $|M|-m)$.

Proposition 5.2 The results of all possible Darboux transformations applied to the operator (20) form one of the 4 sets $D_{ \pm \pm}$described above. These sets consist of $2^{|M|}$ monodromy-free potentials each and are related by the dualities (38), (40). The corresponding Schrödinger equations have $|M|$ quasi-rational solutions of the form

$$
\psi_{i}(x)=R_{i}(x) \exp \left( \pm x^{4} / 4\right)
$$

with some rational functions $R_{i}(x)$. 
Example. We saw above that the operator

$$
L=-D^{2}+x^{6}-7 x^{2}
$$

has two quasi-polynomial eigenfunctions

$$
\psi_{1,2}=\left( \pm \sqrt{2} x^{2}+1\right) \exp \left(-x^{4} / 4\right)
$$

corresponding to $\lambda_{1}=-2 \sqrt{2}$ and $\lambda_{2}=2 \sqrt{2}$ respectively.

The Darboux transformation corresponding to $\psi_{1}$ gives a Schrödinger operator with potential

$$
V_{1}=x^{6}-7 x^{2}-2 D^{2} \ln \psi_{1}=x^{6}-x^{2}+\frac{8 x^{2}-4 \sqrt{2}}{\left(\sqrt{2} x^{2}+1\right)^{2}} .
$$

Note that because $\psi_{1}$ is the ground state of the operator $L$, the result is non-singular on the real line. The corresponding Schrödinger equation

$$
\left(-D^{2}+x^{6}-x^{2}+\frac{8 x^{2}-4 \sqrt{2}}{\left(\sqrt{2} x^{2}+1\right)^{2}}\right) \phi=\lambda \phi
$$

has two quasi-rational solutions:

$$
\phi_{1}=\psi_{1}^{-1}=\frac{1}{\sqrt{2} x^{2}+1} \exp \left(x^{4} / 4\right)
$$

with $\lambda=\lambda_{1}=-2 \sqrt{2}$ and

$$
\phi_{2}=\left(D-D \ln \psi_{1}\right) \psi_{2}=\frac{4 \sqrt{2} x}{\sqrt{2} x^{2}+1} \exp \left(-x^{4} / 4\right)
$$

with $\lambda=\lambda_{1}=-2 \sqrt{2}$. The first function grows at infinity, and thus has no real spectral interpretation, while the second one is a genuine eigenfunction. Because it has a zero at $x=0$ it is the first eigenfunction above the ground state of the transformed potential. Note that the new ground state therefore is not quasi-rational.

Similarly the Darboux transformation corresponding to $\psi_{2}$ gives the potential

$$
V_{2}=x^{6}-7 x^{2}-2 \frac{\mathrm{d}^{2}}{\mathrm{~d} x^{2}} \ln \psi_{2}=x^{6}-x^{2}+\frac{8 x^{2}+4 \sqrt{2}}{\left(\sqrt{2} x^{2}-1\right)^{2}} .
$$

Applying two successive Darboux transformations (which commute) to both $\psi_{1}$ and $\psi_{2}$, we come to the potential

$V_{1,2}=x^{6}-7 x^{2}-2 \frac{\mathrm{d}^{2}}{\mathrm{~d} x^{2}} \ln \left(\mathrm{W}\left(\psi_{1}, \psi_{2}\right)\right)=x^{6}+5 x^{2}-2 \frac{\mathrm{d}^{2}}{\mathrm{~d} x^{2}} \ln \left(\mathrm{W}\left(\sqrt{2} x^{2}+1, \sqrt{2} x^{2}-1\right)\right)$ 


$$
=x^{6}+5 x^{2}-2 \frac{\mathrm{d}^{2}}{\mathrm{~d} x^{2}} \ln (4 \sqrt{2} x)=x^{6}+5 x^{2}+\frac{2}{x^{2}},
$$

which is of course dual to the initial potential $V=x^{6}-7 x^{2}$.

More examples led us to the following

Conjecture. The polynomials $P_{I}(x)$ always have simple zeroes, so all the corresponding potentials $V_{I}$ have the form (12).

We should note that there are monodromy-free potentials which are not of this form, as the following example shows.

Consider a potential of the form

$$
V(x)=x^{6}-\nu x^{2}+\frac{6}{(x-a)^{2}}+\frac{6}{(x+a)^{2}} .
$$

The trivial monodromy conditions give two relations

$$
6 a^{5}-2 \nu a-\frac{3}{2 a^{3}}=0, \quad 10 a^{3}-\frac{3}{8 a^{5}}=0 .
$$

The solution exists only when

$$
\nu= \pm \frac{51}{4 \sqrt{15}}
$$

in which case

$$
a^{8}=\frac{3}{80}
$$

Note that the corresponding $\nu$ is irrational; therefore such a potential can not be a result of Darboux transformations discussed above, It can not even have quasi-rational solutions, as will follow from the results of the next section.

\section{Quasi-rational solutions and Stieltjes rela- tions}

In this section we are going to discuss the relation between two properties: trivial monodromy and the existence of quasi-rational eigenfunctions.

We start with a more general situation. Let $V$ be a rational potential of the form

$$
V(x)=P(x)+\sum_{i=1}^{N} \frac{2}{\left(x-x_{i}\right)^{2}},
$$


where $P(x)$ is a polynomial and $x_{i}$ are distinct. Assume that the corresponding Schrödinger equation

$$
\left(-D^{2}+V(x)\right) \psi=\lambda \psi
$$

has a quasi-rational solution of the form

$$
\psi(x)=\frac{\prod_{j=1}^{K}\left(x-y_{j}\right)}{\prod_{i=1}^{N}\left(x-x_{i}\right)} \exp Q(x),
$$

where $Q(x)$ is another polynomial. The corresponding logarithmic derivative

$$
f(x)=D \ln \psi(x)=\sum_{j=1}^{K} \frac{1}{x-y_{j}}-\sum_{i=1}^{N} \frac{1}{x-x_{i}}+Q^{\prime}(x)
$$

must satisfy the Riccati relation

$$
f^{\prime}+f^{2}=V-\lambda
$$

In particular, the residues at all the poles in the left hand side must be zero. This gives us the following relations between poles $x_{i}$ and zeroes $y_{j}$ of any such eigenfunction

$$
\begin{gathered}
\sum_{j=1}^{K} \frac{1}{x_{k}-y_{j}}-\sum_{i \neq k}^{N} \frac{1}{x_{k}-x_{i}}+Q^{\prime}\left(x_{k}\right)=0, k=1, \ldots, N \\
\sum_{j \neq l}^{K} \frac{1}{y_{l}-y_{j}}-\sum_{i=1}^{N} \frac{1}{y_{l}-x_{i}}+Q^{\prime}\left(y_{l}\right)=0, l=1, \ldots, K
\end{gathered}
$$

We will call these the $\mathbf{S t i e l t j e s}$ relations because in the simplest case, when $Q$ is quadratic and $K=0$, they reduce to the classical Stieltjes relations for the zeroes of Hermite polynomials (see more on this in [6]).

It turns out that these relations are strong enough to imply the trivial monodromy property for the corresponding potential $V(x)$.

Proposition 6.1 If the Schrödinger operator with potential (43) has a quasirational eigenfunction of the form (44) then this operator is monodromy-free.

The proof is elementary (cf. Lemma 2 from [6]). Let

$$
f=-\frac{1}{x-x_{i}}+c_{0}+c_{1}\left(x-x_{i}\right)+c_{2}\left(x-x_{i}\right)^{2}+\ldots
$$


be the Laurent expansion of the logarithmic derivative at a pole $x=x_{i}$. The $i$-th Stieltjes relation (45) means that $c_{0}=0$. We have then

$$
\begin{aligned}
& f^{\prime}+f^{2}=\left(-\frac{1}{x-x_{i}}+c_{1}\left(x-x_{i}\right)+c_{2}\left(x-x_{i}\right)^{2}+\ldots\right)^{\prime}+\left(-\frac{1}{x-x_{i}}+c_{1}\left(x-x_{i}\right)+c_{2}\left(x-x_{i}\right)^{2}+\ldots\right)^{2} \\
& =\frac{2}{\left(x-x_{i}\right)^{2}}-c_{1}+\left(2 c_{2}-2 c_{2}\right)\left(x-x_{i}\right)+\ldots=\frac{2}{\left(x-x_{i}\right)^{2}}-c_{1}+O\left(x-x_{i}\right)^{2} .
\end{aligned}
$$

Since $f^{\prime}+f^{2}=V-\lambda$, we see that the trivial monodromy condition $V_{1}=0$ is satisfied.

Corollary 6.2 The Stieltjes relations imply the locus equations for the corresponding potential $V(x)$ :

$$
-\sum_{i \neq k}^{N} \frac{4}{\left(x_{k}-x_{i}\right)^{3}}+P^{\prime}\left(x_{k}\right)=0, k=1, \ldots, N .
$$

We will give a direct proof of this in the next section where we consider the time-dependent version of the Stieltjes relations.

Note that the corresponding polynomial $P(x)$ is defined here simply as the polynomial part of the rational function

$$
Q^{\prime \prime}(x)+\left(Q^{\prime}\right)^{2}+2 Q^{\prime}\left(\sum_{j=1}^{K} \frac{1}{x-y_{j}}-\sum_{i=1}^{N} \frac{1}{x-x_{i}}\right)
$$

We can be more explicit in the sextic case when $Q(x)= \pm x^{4} / 4$.

Proposition 6.3 Suppose that the Schrödinger operator with potential (43) has a quasi-rational eigenfunction of the form

$$
\psi(x)=\frac{\prod_{j=1}^{K}\left(x-y_{j}\right)}{\prod_{i=1}^{N}\left(x-x_{i}\right)} \exp \epsilon x^{4} / 4,
$$

where $\epsilon= \pm 1$, then polynomial $P(x)$ has the form

$$
P(x)=x^{6}-\epsilon(2 N-2 K-3) x^{2}+2 \epsilon\left(\sum_{j}^{K} y_{j}-\sum_{i}^{N} x_{i}\right) x+\text { const }
$$

In particular the coefficient of the quadratic term

$$
\nu= \pm(2 N-2 K-3)
$$

is an odd integer. 
Indeed, in that case,

$$
\begin{aligned}
2 Q^{\prime} & \left(\sum_{j=1}^{K} \frac{1}{x-y_{j}}-\sum_{i=1}^{N} \frac{1}{x-x_{i}}\right)=2 \epsilon x^{2}\left(\sum_{j=1}^{K}\left(1+\frac{y_{i}}{x}+\frac{y_{i}^{2}}{x^{2}}+\ldots\right)-\sum_{i=1}^{N}\left(1+\frac{x_{i}}{x}+\frac{x_{i}^{2}}{x^{2}}+\ldots\right)\right) \\
& =2 \epsilon(K-N) x^{2}+2 \epsilon\left(\sum_{j}^{K} y_{j}-\sum_{i}^{N} x_{i}\right) x+2 \epsilon\left(\sum_{j}^{K} y_{j}^{2}-\sum_{i}^{N} x_{i}^{2}\right)+O\left(\frac{1}{x}\right) .
\end{aligned}
$$

Note the existence of a linear term

$$
2 \epsilon\left(\sum_{j}^{K} y_{j}-\sum_{i}^{N} x_{i}\right) x,
$$

which does appear already in the simplest case $K=N=1$, as the following example shows.

Example. Consider the quasi-rational eigenfunctions of the form

$$
\psi(x)=\frac{x-y_{1}}{x-x_{1}} \exp -x^{4} / 4
$$

The Stieltjes relations

$$
\frac{1}{x_{1}-y_{1}}-x_{1}^{3}=0, \quad-\frac{1}{y_{1}-x_{1}}-y_{1}^{3}=0
$$

imply that $x_{1}^{3}=y_{1}^{3}$, so $y_{1}=\omega x_{1}$, where

$$
\omega=\frac{-1 \pm \sqrt{3}}{2}
$$

is a root of the equation $\omega^{2}+\omega+1=0$. The pole $x_{1}$ satisfies the equation

$$
x_{1}^{4}=\frac{1}{1-\omega}
$$

and the corresponding potential is

$$
V(x)=x^{6}-3 x^{2}-2(\omega-1) x_{1} x .
$$

The linear term of course disappears in the symmetric case, for then

$$
\sum_{j}^{M} y_{j}=\sum_{i}^{N} x_{i}=0 .
$$


We are going to restrict ourselves to the symmetric case from now on, allowing the additional $\frac{l(l+1)}{x^{2}}$ term at zero:

$$
V(x)=x^{6}-\nu x^{2}+\frac{l(l+1)}{x^{2}}+\sum_{i=1}^{N} \frac{2}{\left(x-x_{i}\right)^{2}} .
$$

The existence of quasi-rational solutions of the form

$$
\psi(x)=x^{\mu} \frac{\prod_{j=1}^{K}\left(x-y_{j}\right)}{\prod_{i=1}^{N}\left(x-x_{i}\right)} \exp \left( \pm x^{4} / 4\right),
$$

with $\mu=-l$ or $\mu=l+1$, leads to the following Stieltjes relations:

$$
\begin{gathered}
\sum_{j=1}^{K} \frac{1}{x_{k}-y_{j}}-\sum_{i \neq k}^{N} \frac{1}{x_{k}-x_{i}} \pm x_{k}^{3}+\frac{\mu}{x_{k}}=0, k=1, \ldots, N \\
\sum_{j \neq l}^{K} \frac{1}{y_{l}-y_{j}}-\sum_{i=1}^{N} \frac{1}{y_{l}-x_{i}} \pm y_{l}^{3}+\frac{\mu}{y_{l}}=0, l=1, \ldots, M .
\end{gathered}
$$

Exactly as before, they imply the locus relations

$$
\sum_{j \neq i}^{N} \frac{2}{\left(x_{i}-x_{j}\right)^{3}}+\frac{l(l+1)}{x_{i}^{3}}+\nu x_{i}-3 x_{i}^{5}=0, \quad i=1, \ldots, N,
$$

with

$$
\nu= \pm(2 N-2 K-2 \mu-3) .
$$

Note that this is true for all values of $l$ (and thus $\mu$ ), not necessarily integers.

The question is whether all solutions of the locus system can be found in such a way. In other words: is it true that any even monodromy-free potential of the form (52) must have a quasi-rational eigenfunction?

The answer is 'No' as the simplest example of the potential

$$
V(x)=x^{6}-(2 l+1) x^{2}+\frac{l(l+1)}{x^{2}}
$$

shows (see section 3 above). A more interesting example is given by

$$
U=x^{6}-x^{2}+2 /(x-a)^{2}+2 /(x+a)^{2} .
$$

The locus relation is

$$
12 a^{8}-4 a^{4}-1=0
$$


which gives us two possibilities: either $a^{4}=1 / 2$ or $a^{4}=-1 / 6$. Since $\nu=$ $\pm(4-2 K-2 \mu-3)= \pm(1-2 K-2 \mu)=1$ and $\mu=0$ or $\mu=1$ one can see that $K$ must be either zero or 1 and we have two possible quasi-rational eigenfunctions:

$$
\psi=\frac{x-b}{x^{2}-a^{2}} \exp \left(-x^{4} / 4\right)
$$

and

$$
\psi=\frac{1}{x^{2}-a^{2}} \exp \left(x^{4} / 4\right)
$$

In the first case the Stieltjes relations are

$$
\frac{1}{a-b}-\frac{1}{2 a}-a^{3}=0, \quad \frac{1}{-a-b}+\frac{1}{2 a}+a^{3}=0,
$$

implying $b=0$ and $a^{4}=1 / 2$. In the second case we have just

$$
-\frac{1}{2 a}+a^{3}=0
$$

which give the same $a^{4}=1 / 2$. Thus we see that the solution $a^{4}=-1 / 6$ of the locus conditions is inconsistent with the Stieltjes relations, and thus the corresponding monodromy-free potential has no quasi-rational eigenfunctions.

We note that the potentials with $a^{4}=1 / 2$ are exactly those given by the Darboux transformations applied to $V=x^{6}-7 x^{2}$ (see example in the section 3 above). This raised the following natural question. Suppose that the monodromy-free potential (52) has a quasi-rational function of the form (53). Is it true that it is a result of Darboux transformation applied to the operators (20) ?

We will show that this answer is negative as well. This will follow from the results of the next section, where we investigate the corresponding Darboux set in more detail.

\section{Geometry and arithmetic of the Darboux set}

By the Darboux set $\mathbf{D}$, we will mean all possible results of Darboux transformations applied to

$$
V=x^{6}-\nu x^{2}+\frac{l(l+1)}{x^{2}} .
$$

It is the union of the sets $D_{ \pm \pm}$described in section 3 . We will allow $l_{0}$ (and the corresponding $\mu$ in (44)) to be arbitrary (not necessarily integer) 
parameters in this section. The corresponding Schrödinger operators will have some monodromy at $x=0$ in that case but will be monodromy-free elsewhere. This does not affect the relation between the locus and Stieltjes algebraic systems shown in the previous section.

We know that the the operator with the potential (57) has a quasi-rational solution (44) only if some of the numbers

$$
M=\frac{ \pm \nu \pm(2 l+1)}{4}
$$

are integers; in that case, as shown in section 3 , the number of such solutions is equal to $|M|$.

It is convenient to represent these cases on the $(l, \nu)$-plane as the grid $\Gamma$ of the lines

$$
\nu \pm(2 l+1)=4 M_{ \pm}
$$

with integer $M$.

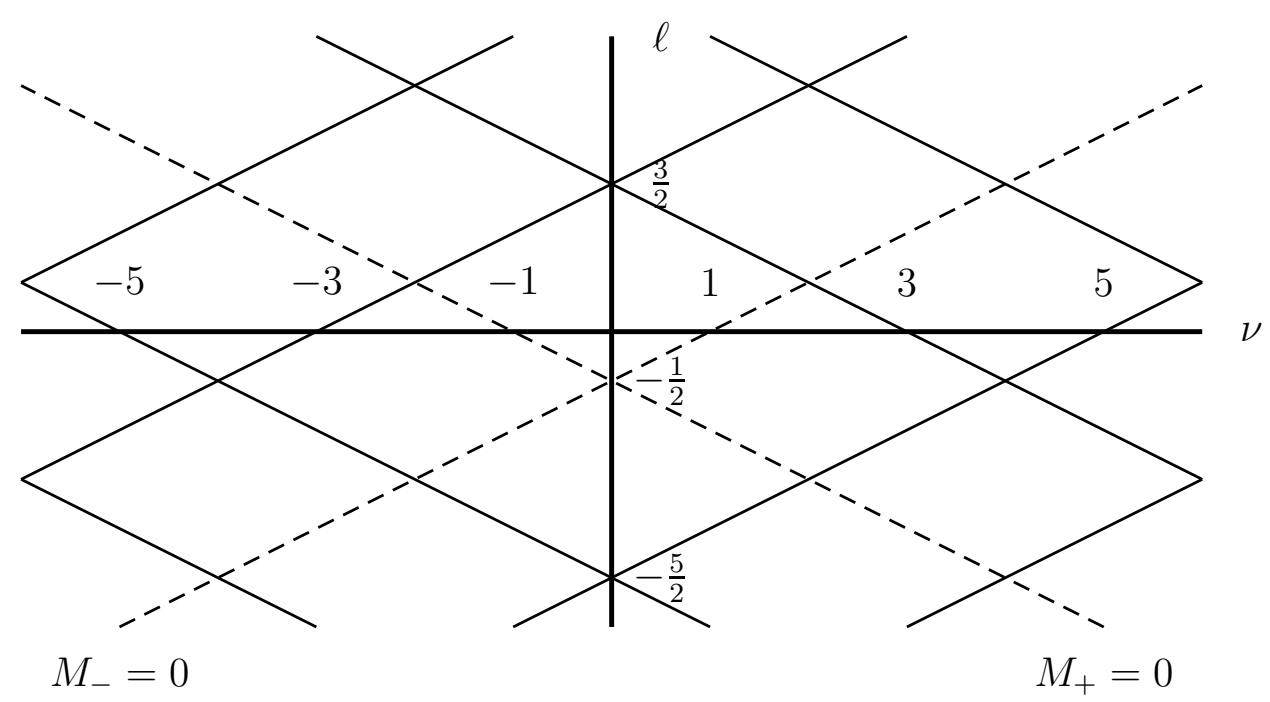

Strictly speaking, when $M_{ \pm}=0$ we do not have quasi-rational solutions but we want anyway to include the corresponding two lines (drawn dashed)

$$
\nu \pm(2 l+1)=0,
$$


which we call special. The number of quasi-rational solutions is proportional to the distance from these lines.

Note also that all the intersection points of the grid have $l$ coordinates which are half-integers. In these cases we can have all four different types of quasi-rational solutions simultaneously, while at all other points in the grid we have only two of them.

Now let us consider the Darboux descendants of these potentials. They have the form

$$
V_{I}=x^{6}-\nu x^{2}+\frac{l(l+1)}{x^{2}}-2 D^{2} \ln P_{2 d}(x)
$$

with the parameters $\nu$ and $l$ different from the original form (57).

Our first claim that these new parameters lie on the same grid $\Gamma$. One can easily check this in all the cases $D_{ \pm \pm}$. For example the Darboux transformations in $D_{+-}$case, change

$$
\nu \rightarrow \nu-6 m, \quad l \rightarrow l+m,
$$

so

$$
M=\frac{\nu-(2 l+1)}{4} \rightarrow \frac{\nu-(2 l+1)}{4}-2 m=M-2 m,
$$

where $m$ is an integer between 1 and $|M|$. So we see that $M$ remain integers, but with modulus $|M|$ decreasing, we moved on the grid closer to the special lines. One can check that the same change

$$
M \rightarrow M-2 m
$$

is true for all other types $D_{ \pm \pm}$as well.

Let us look now at the degree of the corresponding polynomials $P_{2 d}(x)$, which is the same as the number $N$ of the poles in the corresponding potential. We know that in all the cases it has the product form

$$
N=2 d=2 m\left(\left|M_{0}\right|-m\right),
$$

where

$$
M_{0}=\frac{ \pm \nu_{0} \pm\left(2 l_{0}+1\right)}{4}
$$

corresponds to the initial operator

$$
V=x^{6}-\nu_{0} x^{2}+\frac{l_{0}\left(l_{0}+1\right)}{x^{2}} .
$$

This means that the arithmetic of $N$ plays a substantial role here. In particular if the number of poles is $2 p$ where $p$ is prime then this implies 
that corresponding $m$ must be either 1 or $p=\left|M_{0}\right|-1$ (which is actually a dual case to $m=1$, see section 3 ). So we can claim that such a potential must be a result of just one Darboux transformation and the corresponding parameters $(l, \nu)$ must lie on the lines

$$
\nu \pm(2 l+1)=4(p-1) .
$$

At a generic point on these lines the initial parameters $\nu_{0}, \lambda_{0}$ are determined uniquely, so we have exactly $p+1$ such potentials depending on the choice of one of the corresponding $\left|M_{0}\right|=p+1$ quasi-polynomial eigenfunctions.

If $d$ is not prime then the number of Darboux steps $m$ could be any divisor of $d=m k$, where $k$ can be assumed larger than $m$. In that case $\left|M_{0}\right|=k+m$ and we have

$$
\frac{(m+k) !}{m ! k !}
$$

potentials on the lines

$$
\nu \pm(2 l+1)=4(k-m) .
$$

Let us come back now to the question whether any monodromy-free operator with a quasi-rational eigenfunction belongs to the Darboux set or not. The following simple example shows that the answer in general is negative.

Example. We consider the potentials of the form

$$
U=x^{6}-3 x^{2}+2 /(x-a)^{2}+2 /(x+a)^{2}
$$

and check whether they have quasi-rational functions of the form

$$
\psi(x)=\frac{x^{2}-b^{2}}{x^{2}-a^{2}} \exp \left(-x^{4} / 4\right)
$$

with $a$ and $b$ different from zero. The Stieltjes relations here,

$$
\frac{1}{2 b}-\frac{2 b}{b^{2}-a^{2}}-b^{3}=0, \quad-\frac{1}{2 a}+\frac{2 a}{a^{2}-b^{2}}-a^{3}=0,
$$

are equivalent to

$$
a^{4}-b^{4}=1, \quad a^{4}+4 a^{2} b^{2}+b^{4}=0 .
$$

This gives 8 solutions

$$
a^{4}=\frac{1}{1-\epsilon^{2}}, \quad b^{2}=\epsilon a^{2},
$$

where $\epsilon$ satisfies the quadratic equation $\epsilon^{2}+4 \epsilon+1=0$, giving:

$$
\epsilon=-2 \pm \sqrt{3}
$$

Now we claim that the corresponding potentials cannot be the result of Darboux transformations. Indeed in that case $d=1$, so $m$ must be 1 and $\left|M_{0}\right|=2$. On the other hand, since $l=0, \nu=3$ we have $M=\frac{3+1}{4}=1$, which must be equal to $M_{0}-2 m=M_{0}-2$, which is clearly impossible. 


\section{Time-dependent Stieltjes relations and spe- cial solutions of the Calogero-Moser prob- lem}

We now consider the time-dependent Schrödinger equation

$$
i \frac{\partial}{\partial t} \psi=\frac{1}{2}\left(-\frac{\partial^{2}}{\partial x^{2}}+V(x)\right) \psi
$$

If $V(x, t)=x^{6}-\nu x^{2}+\frac{l(l+1)}{x^{2}}$, then this has a linear space of dimension $M$ of quasi-rational solutions, with exponential time-dependence: if $\psi^{i}$ satisfies

$$
\left(-\frac{\partial^{2}}{\partial x^{2}}+V(x)\right) \psi^{i}=\lambda_{i} \psi^{i}
$$

then $\psi_{\mathbf{c}}=\sum_{j=1}^{M} c_{j} \psi^{j} \exp \left(-i \lambda_{i} t / 2\right)$ evidently solves (63). This solution, which has simple but non-trivial time-dependence, may be used, as above, to construct a Darboux transformation

$$
V_{\mathbf{c}_{\mathbf{1}}, \ldots, \mathbf{c}_{\mathbf{M}}}=V-2 \frac{\partial^{2}}{\partial x^{2}} \ln \left(\mathrm{W}_{j=1}^{M}\left(\psi_{\mathbf{c}_{\mathbf{j}}}^{(0)}\right) .\right.
$$

The moving poles of the new potential must thus satisfy the monodromy conditions; in particular they must solve the corresponding Calogero-Moser system.

As an example let us consider the $N=2$ case. Putting exponential time-dependence into the eigenfunctions discussed above, we construct a 2-dimensional space of quasi-polynomial solutions of the time-dependent Schrödinger equation:

$$
i \frac{\partial}{\partial t} \psi=\left(-\frac{\partial^{2}}{\partial x^{2}}+x^{6}-7 x^{2}\right) \psi
$$

namely

$$
\psi_{\mathbf{c}}=\left(c_{1}\left(\sqrt{2} x^{2}+1\right) \mathrm{e}^{i \sqrt{2} t}+c_{2}\left(\sqrt{2} x^{2}-1\right) \mathrm{e}^{-i \sqrt{2} t}\right) \exp \left(-x^{4} / 4\right) .
$$

The Darboux transformation associated with this leads to a new potential

$$
\begin{gathered}
V_{\mathbf{c}}^{(1)}=x^{6}-x^{2}-2 \frac{\partial^{2}}{\partial x^{2}} \ln \left(\psi_{\mathbf{c}}\right) \\
=x^{6}-x^{2}+4 \frac{x^{2}+X^{2}(t)}{\left(x^{2}-X^{2}(t)\right)^{2}},
\end{gathered}
$$


where

$$
\begin{aligned}
X^{2}(t) & =-\frac{c_{1} \mathrm{e}^{i \sqrt{2} t}-c_{2} \mathrm{e}^{-i \sqrt{2} t}}{\sqrt{2}\left(c_{1} \mathrm{e}^{i \sqrt{2} t}+c_{2} \mathrm{e}^{-i \sqrt{2} t}\right)} \\
& =-i \frac{\tan \left(\sqrt{2}\left(t-t_{0}\right)\right)}{\sqrt{2}} .
\end{aligned}
$$

We may easily check in this simple case that the points

$$
\begin{gathered}
x_{1}(t)=X(t), \\
x_{2}(t)=-X(t)
\end{gathered}
$$

indeed satisfy the Calogero-Moser system

$$
\ddot{x}_{i}=\sum_{j=1, j \neq i} \frac{2}{\left(x_{i}-x_{j}\right)^{3}}-3 x_{i}^{5}+x_{i} .
$$

From the locus condition, such a result in fact holds for all potentials of this kind, as will be shown more directly below, where for simplicity we assume the parameter $l=0$.

As in the time-independent case, Darboux transformations take a quasipolynomial solution of the time-dependent Schrödinger equation with an even sextic potential

$$
V^{(0)}(x)=x^{6}-(2 n+3) x^{2}
$$

into a quasi-rational solution

$$
\psi_{\mathbf{c}_{\mathbf{1}}, \ldots, \mathbf{c}_{\mathbf{m}} ; \mathbf{c}_{\mathbf{m}+\mathbf{1}}}^{(m)}=\frac{\mathrm{W}_{j=1}^{m+1}\left(\psi_{\mathbf{c}_{\mathbf{j}}}^{(0)}\right)}{\mathrm{W}_{j=1}^{m}\left(\psi_{\mathbf{c}_{\mathbf{j}}}^{(0)}\right)},
$$

which satisfies the Schrödinger equation with the rational potential

$$
\begin{gathered}
V_{\mathbf{c}_{\mathbf{1}}, \ldots, \mathbf{c}_{\mathbf{m}}}^{(m)}=x^{6}-(2 n+3) x^{2}-2 \frac{\partial^{2}}{\partial x^{2}} \ln \left(\mathrm{W}_{j=1}^{m}\left(\psi_{\mathbf{c}_{\mathbf{j}}}^{(0)}\right)\right. \\
=x^{6}-(2 n+3-6 m) x^{2}-2 \frac{\partial^{2}}{\partial x^{2}} \ln \left(\mathrm{W}_{j=1}^{m}\left(\phi_{\mathbf{c}_{\mathbf{j}}}^{(0)}\right) .\right.
\end{gathered}
$$

Here, as before, the $\phi_{\mathbf{c}_{\mathbf{j}}}^{(0)}$ are the rational factors of the corresponding quasirational solutions $\psi_{\mathbf{c}_{\mathbf{j}}}^{(0)}$.

As before, this solution may be described in terms of its poles and zeroes as

$$
\psi^{(m)}=\frac{\prod_{j=1}^{Z}\left(x-x_{j}\right)^{\alpha_{j}}}{\prod_{k=1}^{P}\left(x-\tilde{x}_{k}\right)^{\beta_{k}}} \exp \left(-x^{4} / 4\right) \exp (i f(t)),
$$


where the multiplicities $\alpha_{j}$ and $\beta_{k}$ are all positive integers. Here the points $x_{k}, \tilde{x}_{k}$ are functions of time, while the time-dependent phase $f(t)$ is not yet determined. We identify the numerator and denominator of the rational part with the polynomial factors of the two Wronskians.

$$
\begin{gathered}
W_{m+1}=\prod_{j=1}^{Z}\left(x-x_{j}\right)^{\alpha_{j}}=\mathrm{W}_{j=1}^{m+1}\left(\phi_{\mathbf{c}_{\mathbf{j}}}^{(0)}\right), \\
W_{m}=\prod_{k=1}^{P}\left(x-\tilde{x}_{k}\right)^{\beta_{k}}=\mathrm{W}_{j=1}^{m}\left(\phi_{\mathbf{c}_{\mathbf{j}}}^{(0)}\right) .
\end{gathered}
$$

We note that the numerator and denominator may have common factors. The Schrödinger equation is now

$$
\begin{gathered}
i \frac{\partial}{\partial t} \frac{W_{m+1}}{W_{m}}-\dot{f} \frac{W_{m+1}}{W_{m}}= \\
\left(-\frac{1}{2}\left(\frac{\partial}{\partial x}-x^{3}\right)^{2}+\frac{1}{2} x^{6}-(N-3 m+3 / 2) x^{2}-\frac{\partial^{2}}{\partial x^{2}} \ln \left(W_{m}\right)\right) \frac{W_{m+1}}{W_{m}}
\end{gathered}
$$

and the Hirota form is hence:

$$
\begin{gathered}
i \frac{\partial W_{m+1}}{\partial t} W_{m}-i W_{m+1} \frac{\partial W_{m}}{\partial t}-\dot{f} W_{m+1} W_{m} \\
+\frac{1}{2}\left(\frac{\partial^{2} W_{m+1}}{\partial x^{2}} W_{m}-2 \frac{\partial W_{m+1}}{\partial x} \frac{\partial W_{m}}{\partial x}+W_{m+1} \frac{\partial^{2} W_{m}}{\partial x^{2}}\right) \\
-x^{3}\left(\frac{\partial W_{m+1}}{\partial x} W_{m}-W_{m+1} \frac{\partial W_{m}}{\partial x}\right) \\
=x^{2}(N-3 m) W_{m+1} W_{m} .
\end{gathered}
$$

Now, on dividing the whole equation by $W_{m} W_{m+1}$, this becomes:

$$
\begin{gathered}
-i \sum_{j=1}^{Z} \frac{\alpha_{j} \dot{x}_{j}}{x-x_{j}}+i \sum_{k=1}^{P} \frac{\beta_{k} \dot{\tilde{x}}_{k}}{x-\tilde{x}_{k}}-\dot{f} \\
+\frac{1}{2}\left(\sum_{j=1}^{Z} \sum_{k=1, k \neq j}^{Z} \frac{\alpha_{j} \alpha_{k}}{\left(x-x_{j}\right)\left(x-x_{k}\right)}-2 \sum_{j=1}^{Z} \sum_{k=1}^{P} \frac{\alpha_{j} \beta_{k}}{\left(x-x_{j}\right)\left(x-\tilde{x}_{k}\right)}+\sum_{j=1}^{P} \sum_{k=1, k \neq j}^{P} \frac{\beta_{j} \beta_{k}}{\left(x-\tilde{x}_{j}\right)\left(x-\tilde{x}_{k}\right)}\right) \\
+x^{3}\left(\sum_{j=1}^{Z} \frac{\alpha_{j}}{x-x_{j}}-\sum_{k=1}^{P} \frac{\beta_{k}}{x-\tilde{x}_{k}}\right)=x^{2}(N-3 m) .
\end{gathered}
$$


Matching coefficients of $x^{2}$, we again get $\sum_{j=1}^{Z} \alpha_{j}-\sum_{j=1}^{P} \beta_{j}=N-3 m$. The coefficients of $x$ must all vanish, by parity, and the coefficient of $x^{0}$ can now be balanced by requiring

$$
-\dot{f}+\sum_{j=1}^{Z} \alpha_{j} x_{j}^{2}-\sum_{j=1}^{P} \beta_{j} \tilde{x}_{k}^{2}=0 .
$$

It remains to check the residues at the zeroes $x_{j}$ and poles $\tilde{x}_{k}$ which give, respectively:

$$
-i \alpha_{j} \dot{x}_{j}+\sum_{k=1, k \neq j}^{Z} \frac{\alpha_{j} \alpha_{k}}{\left(x_{j}-x_{k}\right)}-\sum_{k=1}^{P} \frac{\alpha_{j} \beta_{k}}{\left(x_{j}-\tilde{x}_{k}\right)}+x_{j}^{3} \alpha_{j}=0,
$$

and

$$
i \beta_{k} \dot{\tilde{x}}_{k}-\sum_{j=1, j \neq k}^{Z} \frac{\alpha_{j} \beta_{k}}{\left(\tilde{x}_{k}-x_{j}\right)}+\sum_{j=1, j \neq k}^{P} \frac{\beta_{j} \beta_{k}}{\left(\tilde{x}_{k}-\tilde{x}_{j}\right)}+\tilde{x}_{k}^{3} \beta_{k}=0 .
$$

These are the dynamical Stieltjes relations we seek.

To simplify the notation, if we look at the union of the sets of poles and zeroes, points $z_{i}$, each with a positive or negative exponent $\gamma_{i}$,

$$
\begin{gathered}
z_{i}=x_{i}, \quad \gamma_{i}=\alpha_{i}, \quad i=1, \ldots, Z, \\
z_{i}=\tilde{x}_{i-Z}, \quad \gamma_{i}=-\beta_{i-Z}, \quad i=Z+1, \ldots, Z+P,
\end{gathered}
$$

then this set of relations simplifies:

$$
\dot{z}_{j}=-i \sum_{k=1, k \neq j}^{Z+P} \frac{\gamma_{k}}{\left(z_{j}-z_{k}\right)}-i z_{j}^{3}
$$

It is instructive to differentiate these relations with respect to time. We find:

$$
\ddot{z}_{j}=i \sum_{k=1, k \neq j}^{Z+P} \frac{\gamma_{k}}{\left(z_{j}-z_{k}\right)^{2}}\left(\dot{z}_{j}-\dot{z}_{k}\right)-3 i z_{j}^{2} \dot{z}_{j} .
$$

On substituting (66) into this, we obtain

$$
\begin{array}{r}
\ddot{z}_{j}=i \sum_{k=1, k \neq j}^{Z+P} \frac{\gamma_{k}}{\left(z_{j}-z_{k}\right)^{2}} \\
\left(-i \sum_{l=1, l \neq j}^{Z+P} \frac{\gamma_{l}}{\left(z_{j}-z_{l}\right)}-i z_{j}^{3}+i \sum_{l=1, l \neq k}^{Z+P} \frac{\gamma_{l}}{\left(z_{k}-z_{l}\right)}+i z_{k}^{3}\right) \\
-3 i z_{j}^{2}\left(-i \sum_{l=1, l \neq j}^{Z+P} \frac{\gamma_{k}}{\left(z_{j}-z_{l}\right)}-i z_{j}^{3}\right) .
\end{array}
$$


This may be rearranged as

$$
\begin{gathered}
\ddot{z}_{j}=\sum_{k=1, k \neq j}^{Z+P} \frac{\gamma_{k}\left(\gamma_{k}+\gamma_{j}\right)}{\left(z_{j}-z_{k}\right)^{3}} \\
+\sum_{k=1, k \neq j}^{Z+P} \sum_{l=1, l \neq j, k}^{Z+P} \frac{\gamma_{k}}{\left(z_{j}-z_{k}\right)^{2}}\left(\frac{\gamma_{l}}{\left(z_{j}-z_{l}\right)}+z_{j}^{3}-\frac{\gamma_{l}}{\left(z_{k}-z_{l}\right)}-z_{k}^{3}\right) \\
-3 z_{j}^{2}\left(\sum_{l=1, l \neq j}^{Z+P} \frac{\gamma_{k}}{\left(z_{j}-z_{l}\right)}-i z_{j}^{3}\right) .
\end{gathered}
$$

This further simplifies, after some elementary manipulation, to

$\ddot{z}_{j}=\sum_{k=1, k \neq j}^{Z+P} \frac{\gamma_{k}\left(\gamma_{k}+\gamma_{j}\right)}{\left(z_{j}-z_{k}\right)^{3}}-3 z_{j}^{5}-\sum_{k=1, k \neq j}^{Z+P} \sum_{l=1, l \neq j, k}^{Z+P} \frac{\gamma_{k} \gamma_{l}}{\left(z_{j}-z_{k}\right)\left(z_{j}-z_{l}\right)\left(z_{k}-z_{l}\right)}-\sum_{k=1, k \neq j}^{Z+P} \gamma_{k}\left(2 z_{j}+z_{k}\right)$.

Now in this long expression the double sum

$$
\sum_{k=1, k \neq j}^{Z+P} \sum_{l=1, l \neq j, k}^{Z+P} \frac{\gamma_{k} \gamma_{l}}{\left(z_{j}-z_{k}\right)\left(z_{j}-z_{l}\right)\left(z_{k}-z_{l}\right)}
$$

is identically zero. The final term

$$
\sum_{k=1, k \neq j}^{Z+P} \gamma_{k}\left(2 z_{j}+z_{k}\right)
$$

is equal to

$$
\left.z_{j}\left(2 \sum_{k=1}^{Z+P} \gamma_{k}-3 \gamma_{j}\right)+\sum_{k=1}^{Z+P} \gamma_{k} z_{k}\right)
$$

In the special case where all zeroes and poles are simple, $\gamma_{k}= \pm 1$, and $\sum_{k=1}^{Z+P} \gamma_{k}=Z-P$, so this term becomes $z_{j}\left(2(Z-P)-3 \gamma_{j}\right)$. Further, from the quadratic term above we saw $Z-P=N-3 m$. The term $\sum_{k=1}^{Z+P} \gamma_{k} z_{k}$ is supposed to be identically zero by parity.

Thus, remarkably, the system decouples, giving:

$$
\ddot{x}_{j}=\sum_{k=1, k \neq j}^{Z} \frac{2}{\left(x_{j}-x_{k}\right)^{3}}-3 x_{j}^{5}-x_{j}(2(Z-P)-3) .
$$

for the zeroes, and

$$
\ddot{\tilde{x}}_{j}=\sum_{k=1+Z, k \neq j}^{Z+P} \frac{2}{\left(\tilde{x}_{j}-\tilde{x}_{k}\right)^{3}}-3 \tilde{x}_{j}^{5}-\tilde{x}_{j}(2(Z-P)+3) .
$$

for the poles. 
Proposition 8.1 The Stieltjes relations 64, 65) with $\alpha_{i}=\beta_{j}=1$ imply that the zeroes and poles of solutions of the non-stationary Schrödinger equation satisfy the uncoupled Calogero-Moser systems in different sextic potentials with the Hamiltonians

$$
H=\frac{1}{2} \sum_{i=1}^{Z} p_{i}^{2}+\frac{1}{2} \sum_{i=1}^{Z} x_{i}^{6}-\frac{1}{2} \sum_{i=1}^{Z}(2(Z-P)-3) x_{i}^{2}+\frac{1}{2} \sum_{i=1}^{Z} \sum_{j=1, j \neq i}^{Z} \frac{1}{\left(x_{i}-x_{j}\right)^{2}}
$$

for the zeroes, and

$$
\tilde{H}=\frac{1}{2} \sum_{i=1}^{P} \tilde{p}_{i}^{2}+\frac{1}{2} \sum_{i=1}^{P} \tilde{x}_{i}^{6}-\frac{1}{2} \sum_{i=1}^{P}(2(Z-P)+3) \tilde{x}_{i}^{2}+\frac{1}{2} \sum_{i=1}^{P} \sum_{j=1, j \neq i}^{P} \frac{1}{\left(\tilde{x}_{i}-\tilde{x}_{j}\right)^{2}}
$$

for the poles.

Thus the first order equations (66) again imply that the simple poles and zeroes satisfy separate Calogero-Moser systems, in even sextic potentials, but with different $O\left(x^{2}\right)$ terms. We may thus regard (66) as being a Bäcklund transformation for Calogero-Moser systems of this type. This does not imply the system is integrable however, for the Bäcklund transformation does not depend on an arbitrary constant. Further, it can only be iterated finitely many times.

Alternatively, the system of dynamical Stieltjes relations may be understood as a canonical transformation. Taking all the poles and zeroes to be simple, if the momentum variable conjugate to the zero $x_{j}$ is $p_{j}$, and that conjugate to $\tilde{x}_{j}$ is $\tilde{p}_{j}$, the relations read:

$$
p_{j}=-i \sum_{k=1, k \neq j}^{Z} \frac{1}{\left(x_{j}-x_{k}\right)}+i \sum_{k=1}^{P} \frac{1}{\left(x_{j}-\tilde{x}_{k}\right)}-i x_{j}^{3},
$$

and

$$
\tilde{p}_{k}=i \sum_{j=1}^{Z} \frac{1}{\left(\tilde{x}_{k}-x_{j}\right)}-i \sum_{j=1, j \neq k}^{P} \frac{1}{\left(\tilde{x}_{k}-\tilde{x}_{j}\right)}-i \tilde{x}_{k}^{3}=0 .
$$

If we define

$$
\begin{array}{r}
S=-i \log \left(\frac{\prod_{j=1}^{Z} \prod_{k=1, k>j}^{Z}\left(x_{j}-x_{k}\right) \prod_{m=1}^{P} \prod_{n=1, n>m}^{P}\left(\tilde{x}_{m}-\tilde{x}_{n}\right)}{\prod_{j=1}^{Z} \prod_{k=1, k \neq j}^{P}\left(x_{j}-\tilde{x}_{k}\right)}\right) \\
-i \sum_{j=1}^{Z} \frac{x_{j}^{4}}{4}+i \sum_{k=1}^{P} \frac{\tilde{x}_{j}^{4}}{4},
\end{array}
$$


then the Stieltjes relations (69, 70) become:

$$
\begin{gathered}
p_{j}=\frac{\partial S}{\partial x_{j}}, \\
\tilde{p}_{j}=-\frac{\partial S}{\partial \tilde{x}_{j}} .
\end{gathered}
$$

They are thus seen to give a canonical transformation with generating function $S$. Note that dropping $i$ in these formulae corresponds to a change of sign in the potential and thus to an attractive, rather than repulsive, pairwise potential.

This transformation is straightforward when the numbers $Z$ and $P$ of zeroes and poles are equal, but otherwise we may still understand it as generating a mapping from simple solutions of a Calogero-Moser system, with few degrees of freedom, to more complicated solutions, with more degrees of freedom - of course such solutions will be special, with some algebraic relations between the new coordinates and momenta. The rational potentials constructed above correspond to the most special ones of all these, which are connected by a finite chain of such canonical transformations to the 'empty' Calogero-Moser system with no particles at all. Since it is elementary to show that this transformation preserves the Hamiltonian, $H=\tilde{H}$, all the solutions of Calogero-Moser systems constructed here, related by finitely many such transformations to one with no degrees of freedom, must have zero energy. We believe that the same should be true for other Inozemtsev integrals as well.

One immediate consequence of the fact that the dynamical Stieltjes relations imply that the simple poles and zeroes separately satisfy CalogeroMoser equations is that the analogous stationary Stieltjes relations must imply that the poles and zeroes satisfy stationary Calogero-Moser equations in the appropriate potentials; these are precisely the locus conditions for these potentials.

\section{Concluding remarks}

We have seen that the monodromy-free potentials with sextic growth are described by the algebraic locus equations and are related to equilibria of the corresponding version of the Calogero-Moser problem, which is known to be integrable [8]. So, is the locus system (56) 'integrable'? In which sense ?

We have shown that Darboux transformations are not enough to describe the whole locus, even if we assume the existence of quasi-rational solutions. The geometry and arithmetic of the corresponding potentials seems to be 
quite interesting as well as the algebraic geometry of the corresponding (zerodimensional) affine varieties. In particular, the Stieltjes relations can be considered as correspondences (in the sense of algebraic geometry) between two different locus varieties, which is a partially defined multivalued algebraic map. Although they can be considered as natural analogues of the Bäcklund transformations their existence is not enough for 'integrability' and holds for any growth at infinity (see section 6 above).

But the general structure of the sextic locus still remain largely unclear. In relation with Inozemtsev's results [8] we note that the general solution of the Calogero-Moser system in a sextic potential is given in terms of some matrix analogues of the elliptic functions and are expressed in terms of the high genus Riemann $\theta$-functions. This means that an elementary description of all corresponding equilibria seems to be unlikely in agreement with our result.

In that sense the situation looks similar to the case of the elliptic potentials studied by Gesztesy and Weikard [21, who showed that all the corresponding potentials must be finite-gap in the sense of [22]. The problem of finding an effective description of the elliptic finite-gap potentials was first raised by S.P. Novikov in the 70s, who was inspired by the first examples found in his work with Dubrovin [23]. This area was revitalised after the appearance of the famous Trebich-Verdier preprint [24], which showed that there are examples, which can not be found using the KdV flows from the classical Lamé potentials

$$
V(x)=m(m+1) \wp(x),
$$

where $\wp(x)$ is the Weierstrass elliptic function. In spite of the numerous efforts in this direction (see e.g. Enolskii-Eilbeck [25], Taimanov [26], Smirnov [27]) the problem seems to be far from a satisfactory solution.

Our sextic problem can be considered as a simple model for this important problem and thus deserves further investigation. It could also very interesting from the analytic-differential point of view, see the recent paper [28] by Eremenko, Gabrielov and Shapiro.

\section{Acknowledgements}

This work has been partially supported by the European Union through the FP6 Marie Curie RTN ENIGMA (Contract number MRTN-CT-20045652). We have also received support from the European Science Foundation through the MISGAM programme. 
The work of A.V. was also partially supported by the EPSRC (grant $\mathrm{EP} / \mathrm{E} 004008 / 1)$.

\section{References}

[1] Duistermaat, J.J., Grünbaum, F.A., Commun. Math. Phys., 103, 177240,(1986).

[2] J.L.Burchnall, and T.W.Chaundy , Proc. London Math. Soc. 30, (192930), 401-414.

[3] Adler, M., Moser, J., Commun. Math. Phys., 61, 1-30, (1978).

[4] H.Airault, H.Mc Kean, J.Moser, Comm. Pure Appl.Math., 30 (1977), 95-148.

[5] Oblomkov, A.A., Theor. Mat. Phys., 121, 1574-84, (1999).

[6] Veselov, A.P., J. Phys A.: Math and Gen., 34, 3511-3519, (2001).

[7] Veselov, A.P. and Shabat, A.B., Functional Analysis and its Applications, 27(2), 81-96, (1993).

[8] Inozemtsev, V.I., Functional Analysis and its Applications, 23, 81-82, (1989).

[9] A. Turbiner, Sov. Phys. JETP, 67, 230 (1988).

[10] Turbiner A.V., Commun. Math. Phys., 118, 467-474, (1988).

[11] Chhajlany, S.C., Malnev, V.N., J. Phys. A23, 3711-3718 (1990).

[12] Chhajlany, S.C., Letov, D.A., Malnev, V.N., J. Phys. A24, 2731-2741 (1991).

[13] Tater, M., Turbiner A.V., J. Phys.A26, 697-710, (1993).

[14] C.M. Bender, G.V. Dunne Quasi-Exactly Solvable Systems and Orthogonal Polynomials Journal of Mathematical Physics, 37, 6-11, (1996).

[15] G. Krylov and M. Robnik, J. Phys. A: Math. Gen. 34, 26, 5403-5415, (2001).

[16] P. Dorey, C. Dunning, R. Tateo, J. Phys. A: Math. Gen. 34, 5679-5704, (2001). 
[17] G. Darboux, C.R.Acad.Sci.,Paris 94, p.1456 (1882).

[18] M. M. Crum, Quart. J. Math. Oxford 6, 121, (1955).

[19] Stieltjes, T.J., C.R. Acad. Sci. Paris, 100, 439-440, (1885).

[20] Stieltjes, T.J., C.R. Acad. Sci. Paris, 100, 620-622 (1885).

[21] F. Gesztesy and R. Weikard, Acta Math., 176, 73-107 (1996).

[22] B.A. Dubrovin, V.B. Matveev and S.P. Novikov, Russian Math. Surveys, 31, 59-146, (1976).

[23] B.A. Dubrovin, S.P. Novikov, Soviet Phys. JETP 67, 1058-1063, (1974).

[24] A. Treibich and J.-L. Verdier, Solitons elliptiques, in The Grothendieck Festschrift, Volume III, P. Cartier, L. Illusie, N. M. Katz, G. Laumon, Y. Manin and K. A. Ribet (eds.), Birkhuser, Basel, 1990, 437-480.

[25] Enolskii V.Z., Eilbeck J.C., J. Phys. A: Math. Gen., 28, 1069-1087, (1995).

[26] I.A. Taimanov, Acta Math. Appl. 36, 119-124, (1994).

[27] A.O. Smirnov, Acta Math. Appl., 36, 31-55, (1994).

[28] A. Eremenko, A. Gabrielov, B. Shapiro, Ann. Inst. Fourier, 58, 603-624 (2008). 